0093-6413(95)00030-5

\title{
QUADRATIC HAMILTONIANS ON THE UNIT SPHERE
}

Jörg Frauendiener

Max-Planck-Institut für Astrophysik, Karl-Schwarzschild-Straße 1, D-85740 Garching, Germany

(Received 4 January 1995; accepted for print 15 March 1995)

\section{Introduction}

The purpose of this letter is to point out the general form of a certain class of Hamiltonians on the unit sphere. We consider Hamiltonians as functions of the cartesian coordinates $(x, y, z)$ in terms of which the unit sphere is given by the equation $x^{2}+y^{2}+z^{2}=1$ The class of Hamiltonians treated is then defined as those functions which are at most quadratic in these coordinates. Such Hamiltonians occur naturally in various problems of mechanics $[1-3]$, optics [4] and others. In section 2. we introduce the necessary machinery and apply it in section 3. to obtain the classification of quadratic Hamiltonians on the unit sphere This extends the work in [5] where a classification of biparametric quadratic Hamiltonans has been given.

\section{Preliminaries}

Let $S^{2}$ be given as the set of unit vectors in $\mathbb{R}^{3}, S^{2}=\left\{x^{a}: x^{a} x^{b} \eta_{a b}=1\right\}$, where $\eta_{a b}=$ $\operatorname{diag}(1,1,1)$ is the standard metric in $\mathbb{R}^{3}$ and $x^{a}=(x, y, z)$. Define the function $S\left(x^{a}\right)=$ $\eta_{a b} x^{a} x^{b}-1$, then $S^{2}$ is the zero-set of $S$. Since it is a two-dimensional manifold all symplectic structures on the sphere are proportional to the area form of $S^{2}$ which we take as the symplectic form $\Omega$ on $S^{2}$. So $\Omega=x d y \wedge d z+y d z \wedge d x+z d x \wedge d y=\frac{1}{2} \epsilon_{a b c} x^{a} d x^{b} \wedge d x^{c}$. 
Here $\epsilon_{a b c}$ is the alternating Levi-Cività symbol. The Poisson algebra of functions on the sphere with respect to this symplectic form is generated by the Poisson brackets between the coordinate functions

$$
\{x, y\}=z, \quad\{y, z\}=x, \quad\{z, x\}=y
$$

We now define the class $\mathcal{Q}$ of functions on $S^{2}$ which are at most quadratic in the coordinates $(x, y, z)$. Hence $H \in \mathcal{Q}$ if and only if $H=\frac{1}{2} A_{a b} x^{a} x^{b}+B_{a} x^{a}+D$ with $A_{a b}$ a constant quadratic form, $B_{a}$ a constant (co)vector and $D$ an arbitrary constant. Our aim is to find a standard form for Hamiltonians in $\mathcal{Q}$. To this end we regard Hamiltonians as equivalent if they induce the same phase diagram, i.e., if the trajectories of their hamiltonian vector fields can be mapped onto each other by symplectic transformations, where we do not care about the parametrization. Hence, two Hamiltonians are equivalent if there exists a symplectic transformation which maps one hamiltonian vector field onto a multiple of the other Thus, the first question to be answered is, how big is the group of symplectic transformations which leave $\mathcal{Q}$ invariant. We first consider infinitesimal transformations. Let $X=X \partial_{c}$ be a vector field in $\mathbb{R}^{3}$, where $X^{c}\left(x^{a}\right)$ are three functions on $\mathbb{R}^{3}$ and $\partial_{c}:=\frac{\partial}{\partial x^{c}}$. This vector field will be tangent to the unit sphere iff $L_{X} S \propto S, L_{X}$ being the Lie derivative along the vector field $X$. This implies $\eta_{a b} x^{a} X^{b}=\alpha S$ for some function $\alpha$. From this we get that $X$ can be represented on $S^{2}$ in the form

$$
X=\epsilon^{a b c} x_{a} B_{b} \partial_{c}
$$

for three arbitrary functions $B_{c}$. Here, the symbol $\epsilon^{a b c}$ denotes the "dual" Levi-Cività symbol which is defined by the equation $\epsilon_{a b c} \epsilon^{a b c}=6$. Note, that $X$ vanishes on $S^{2}$ if $B_{c}=B x_{c}$, so there are only essentially two free functions contained in $X$ as it should be for vector fields on a two-dimensional manifold. Next, we ask which of these vector fields leave $\mathcal{Q}$ invariant. This entails the condition $L_{X} \mathcal{Q} \subset \mathcal{Q}$. Let $H \in \mathcal{Q}$ then

$$
L_{X} H=A_{a b} x^{a} X^{b}+B_{a} X^{a} .
$$

This will be in $\mathcal{Q}$ iff $X^{a}$ is at most linear $x^{a}$ which, in view of (2.2) implies that $B_{a}$ has to be constant. Hence, there exists a three-dimensional space of vector fields on $S^{2}$ which leaves $\mathcal{Q}$ invariant. It is easy to see that this space is generated by the three 
infinitesimal rigid rotations around three orthogonal axes in $\mathbb{R}^{3}$. Hence, the group which leaves $\mathcal{Q}$ invariant is locally isomorphic to the group $S O(3)$ of proper rotations. The group $O(3)$ (also locally isomorphic to $S O(3)$ ) additionally includes reflections and also leaves $\mathcal{Q}$ invariant. However, while all proper rotations are also symplectic transformations (they leave $\Omega$ invariant), this is not so for reflections, because they map $\Omega$ to $-\Omega$. Hence, the subgroup of symplectic transformations leaving $\mathcal{Q}$ invariant is exactly the group $S O(3)$. This has been pointed out already in [5].

\section{The standard form}

As we mentioned above we consider Hamiltonians as equivalent iff their hamiltonian vector fields can be mapped onto multiples of each other by symplectic transformations. This implies that any $H \in \mathcal{Q}$ is equivalent to

$$
H+a S, \quad b H . \quad H+c, \quad R^{*} H,
$$

where $a, b, c \in \mathbb{R}, b \neq 0$ and $R \in S O(3)$. There are ten parameters in $H$ ( $\operatorname{six}$ in $A_{a b}$, three in $B_{a}$ and one in $C$ ) and alltogether there are six parameters in the equivalence transformations (3.1). So we expect that we will be left with four essential parameters in the standard form for $H$.

Let us start with the simplest case: $A_{a b}=0$ and $B_{a}=0$. Then $H$ is constant and thus equivalent to zero: $H \sim 0$. Now let $B_{a}$ be non-vanishing. Then we can use the rotational freedom to align $B^{a}$ with the $z$-axis and the scaling transformation to achieve $H \sim$ :

Let $A_{a b} \neq 0$. Then $H=\frac{1}{2} A_{a b} x^{a} x^{b}+B_{a} x^{a}+C$. Let $\lambda_{1}, \lambda_{2}, \lambda_{3}$ be the three eigenvalues of $A^{a}{ }_{b}$. We first assume that these are all equal. Then $A_{a b}$ is diagonal and by adding an appropriate multiple of $S$ we see that $A_{a b} x^{a} x^{b}$ is equivalent to a constant and hence to zero. So this brings us back to the cases already discussed. Now if $\lambda_{1}=\lambda_{2} \neq \lambda_{3}$ then we can use part of the rotational freedom to put $A_{a b}$ into diagonal form. Thus $A_{a b} x^{a} x^{b}=\lambda_{1}\left(x^{2}+y^{2}\right)+\lambda_{3} z^{2}$. Since we can still perform rotations around the $z$-axis we can use these to orient the $x$-axis so that $B_{a}$ is in the $x z$-plane. Finally, using the remaining equivalence transformations we find that $H \sim \frac{1}{2} z^{2}+A x+C z$. 
In the last case all the eigenvalues are different. Thus, putting $A_{a b}$ into diagonal form uses up all the rotational freedom apart from even permutations of the three axes. The effect of the equivalence transformations on the eigenvalues is

$$
\lambda_{i} \mapsto \lambda_{i}+a, \quad \lambda_{i} \mapsto b \lambda_{i}
$$

We can use the additive tranformation to put one of the eigenvalues equal to zero. Which of these we choose depends on the relation between $B_{a}$ and the eigenvectors of $A_{a b}$. Let us first assume that $B_{a}$ is proportional to an eigenvector which we can choose (by way of an even permutation) as pointing along the $z$-axis. Then we use the shift to put $\lambda_{3}=0$ and the scaling transformation to put $\lambda_{\mathbf{I}}=1$. Thus we have found that $H \sim \frac{1}{2} x^{2}+\frac{1}{2} \lambda y^{2}+C z$. Now, if $B_{a}$ is in the subspace spanned by two eigenvectors then we can choose those as being the $x$ - and the $y$-axis. Again. shifting $\lambda_{3}$ to zero and dividing by $\lambda_{1}$ (which is non-zero) we obtain the equivalence $H \sim \frac{1}{2} x^{2}+\frac{1}{2} \lambda y^{2}+A x+B y$.

The final case is where $B_{a}$ is generic, i.e., its components along the eigenvectors are all non-zero. Then we can again put $\lambda_{3}$ to zero and scale $\lambda_{1}$ to unity to obtain $H \sim \frac{1}{2} x^{2}+$ $\frac{1}{2} \lambda y^{2}+A x+B y+C z$.

To summarize then, we have found the following classification

\begin{tabular}{|cc|c|}
\hline$A_{a b}$ & $B_{a}$ & $H$ \\
\hline$(3)$ & {$[0]$} & 0 \\
$(3)$ & {$[3]$} & $\tilde{z}$ \\
$(21)$ & {$[3]$} & $\frac{1}{2} x^{2}+A x+C z$ \\
$(111)$ & {$[1]$} & $\frac{1}{2} x^{2}+\frac{1}{2} \lambda y^{2}+C z$ \\
$(111)$ & {$[2]$} & $\frac{1}{2} x^{2}+\frac{1}{2} \lambda y^{2}+A x+B y$ \\
$(111)$ & {$[3]$} & $\frac{1}{2} x^{2}+\frac{1}{2} \lambda y^{2}+A x+B y+C z$ \\
\hline
\end{tabular}

Here we have indicated in the column below $A_{a b}$ the degeneracies of the spectrum of $A_{a b}:(111),(21)$ and $(3)$ indicating that $A_{a b}$ has three, two or one different eigenvalue( $s$ ). Similarly, $[n]$ in the column below $B_{a}$ indicates that $B_{a}$ lies in a subspace spanned by $n$ eigenvectors of $A_{a b}$.

\section{Conclusion}

In this article we have completed the work started in Ref. [5] on the classification of 
quadratic Hamiltonians on the unit sphere. The basic tool for this was the geometry underlying the structure of the unit sphere as a symplectic manifold. It would be useful to have the phase portraits for the various classes and to discuss the bifurcations which might occur. This will be done in some further article.

References

[1] A. Deprit and A. Elipe, Celest. Mech. 51 227-250, (1991)

[2] B. Miller, Celest. Mech. 51 251-270 (1991)

[3] A. Elipe and V. Lanchares, Bol. Astron. Observ. Madrid $1256-67$ (1990)

[4] D. David, D.D. Holm and M. V. Tratnik, Phys. Rep. 187 281-367 (1990)

[5] A. Elipe and V. Lanchares, Mech. Res. Comm. 21 209-214 (1994) 